Mitteilungen der Österreichischen Geographischen Gesellschaft, 158. Jg. (Jahresband), Wien 2016, S. 359-364

\title{
29. Sitzung der Expertengruppe der Vereinten Nationen FÜR GEOGRAPHISCHE NAMEN BangKoK [Krung TheP], 25.-29. April 2016
}

\author{
Peter JORDAN, Wien* \\ mit 3 Abb. im Text
}

\section{Nationale und internationale Standardisierung geographischer Namen}

Die Expertengruppe der Vereinten Nationen für geographische Namen (United Nations Group of Experts on Geographical Names, UNGEGN) wurde 1959/1960 im Zuständigkeitsbereich des Economic and Social Council (ECOSOC) der Vereinten Nationen eingerichtet und fungiert seither als interdisziplinär zusammengesetztes Fachgremium vor allem von Geographen, Kartographen, Geodäten, Linguisten und Historikern. Es berät Empfehlungen zur Verwendung und Standardisierung geographischer Namen, die dann von den Konferenzen der Vereinten Nationen zur Standardisierung geographischer Namen (United Nations Conferences on the Standardization of Geographical Names, UNCSGN) nochmals geprüft und gegebenenfalls beschlossen und in den Rang von Resolutionen der Vereinten Nationen erhoben werden.

Die Standardisierung und angemessene Verwendung geographischer Namen ist den Vereinten Nationen nicht nur deshalb ein Anliegen, weil eindeutige geographische Namen für die sichere Orientierung und die Georeferenzierung von geographischen Objekten wichtig sind, sondern auch weil geographische Namen eine starke symbolische Wirkung haben, für raumbezogene Identitäten stehen, emotionale Bindungen zu Orten unterstützen und damit auch Konfliktpotenzial in sich bergen. Der (mittlerweile ausgestandene) Streit um die Kärntner Ortstafeln, die immer wieder aufflammende Kontroverse um die italienischen Namen in Südtirol [Südtirol/Alto Adige], der hartnäckige Widerstand Griechenlands gegen den Namen Makedonien als Bezeichnung für den Nachfolgestaat Jugoslawiens oder der Nachdruck, mit welchem sich die Republik Korea und Japan für die internationale Verwendung ihres jeweiligen Namens für das Meer zwischen beiden Staaten einsetzen (Japanisches Meer oder Ostmeer), sind beredte Beispiele dafür. Die Standardisierung und geregelte Verwendung von geographischen Namen dient also auch der Konfliktprävention und Konfliktlösung oder sollte zumindest diese Aufgabe erfüllen und ist daher den Vereinten Nationen wichtig.

Österreich hat sich in dieser Expertengruppe von Anfang an stark engagiert und Josef Breu, später auch Präsident der Österreichischen Geographischen Gesellschaft (ÖGG), zählte zu ihren Mitbegründern. Er richtete im Jahr 1969 nach den Empfehlungen der Vereinten Nationen auch die Abteilung (heute: Arbeitsgemeinschaft) für Kartographische Ortsnamenkunde (AKO) als natio-

\footnotetext{
* Hofrat Prof. h.c. Univ.-Doz. Dr. Peter Jordan, Vorsitzender der Arbeitsgemeinschaft für Kartographische Ortsnamenkunde (AKO), Convenor der UNGEGN Working Group on Exonyms, Institut für Stadt- und Regionalforschung, Österreichische Akademie der Wissenschaften, Postgasse 7/4/2, A-1010 Wien; E-Mail: peter.jordan@oeaw.ac.at, http://www.oeaw.ac.at/isr
} 
nales österreichisches Standardisierungsgremium ein, die der Österreichischen Kartographischen Kommission (ÖKK) in der ÖGG zugehört, aber auch „,in Verbindung“ mit der Österreichischen Akademie der Wissenschaften (ÖAW) steht. Der Austausch zwischen AKO (wie auch den Standardisierungsgremien anderer Länder) und der Ebene der Vereinten Nationen erfolgt in beide Richtungen: Die AKO versucht, Empfehlungen der Vereinten Nationen in Österreich umzusetzen oder auf österreichische Verhältnisse anzuwenden, bringt ihrerseits aber auch österreichische Ideen und Vorschläge auf UNO-Ebene ein. So ergänzen sich internationale und nationale Standardisierung.

Beide Ebenen sind (jedenfalls in Österreich) nicht nur institutionell, sondern auch in Personalunion verbunden, was den erwähnten wechselseitigen Austausch erleichtert: Der Vorsitzende der AKO ist zugleich der von der ÖAW nominierte und von der österreichischen Bundesregierung delegierte Leiter der österreichischen Delegation bei den UNGEGN-Sitzungen und in den UNCSGN, sein Stellvertreter ist jedenfalls ein weiteres Mitglied der österreichischen Delegation, wobei auch noch andere AKO-Mitglieder mitentsandt werden können. Die beiden Vorsitzenden der AKO besetzen derzeit außerdem zwei von zwölf Positionen der operativen Leitung der UNGEGN, nämlich die des Convenors der Working Group on Exonyms (Peter JoRDAN seit 2007) und die des internationalen Koordinators der Toponymic Guidelines for Map and Other Editors (Gerhard RAMPL seit 2014).

\section{Die Plenarsitzung in Bangkok}

Die 29. Sitzung der UNGEGN folgte der Sitzung des Jahres 2014 in New York und fand diesmal ausnahmsweise in Bangkok [Krung Thep] im dortigen Konferenzgebäude der Vereinten Nationen statt $(\text { Abb. 1, 2) })^{1}$ - in der Absicht asiatischen Ländern die Teilnahme zu erleichtern. Tatsächlich stellten unter den 180 Teilnehmern asiatische Länder die größten Delegationen: Thailand mit 20, Indonesien mit 18, die Republik Korea mit 13, und auch Japan und China waren stark vertreten. Allerdings fehlten viele Länder des östlichen und südlichen Europas sowie Lateinamerikas. Selbst die Vereinigten Staaten von Amerika hatten nur einen Delegierten entsandt. Österreich war durch den Vorsitzenden der AKO, Peter JoRDAN, und durch seinen Stellvertreter, den Linguisten Gerhard RAMPL, vertreten.

Von den insgesamt 88 schon geraume Zeit vor Beginn der Sitzung eingereichten und auf der UNGEGN-Homepage einsehbaren Arbeitspapieren (working papers), von denen in der Sitzung selbst nicht alle präsentiert, sondern teilweise nur zur Diskussion gestellt wurden, stammten neun aus Österreich. Drei davon stellten österreichische namenkundliche Projekte vor. Eines präsentierte das vom Institut für Corpuslinguistik und Texttechnologie der ÖAW seit 1989 herausgegebene und 2014 abgeschlossene, maßgeblich von Isolde HAUSNER betreute Altdeutsche Namenbuch mit dem Untertitel „Die Überlieferung der Ortsnamen in Österreich und Südtirol von den Anfängen bis 1200"; ein zweites den von den Salzburger Linguisten Ingo ReIFFENSTEIN und Thomas LindNER herausgegebenen und 2015 erschienenen ersten Band (Lungau) des als fünfbändig geplanten Historisch-Etymologische Lexikons der Salzburger Ortsnamen (HELSON); ein drittes das federführend vom Urban-Jarnik-Institut in Klagenfurt a.W. (Martina PIKo-Rustia) betreute Projekt der Erhebung und Dokumentation slowenischer Flur- und Hofnamen im südlichen Teil Kärntens, welche von der UNESCO in die nationale Liste des immateriellen kulturellen Erbes aufgenommen worden sind.

Die anderen sechs Arbeitspapiere waren von den beiden österreichischen UNGEGN-Delegierten in Ausübung ihrer UNGEGN-Funktionen ausgearbeitet worden. Drei von Peter JORDAN verfasste stellten jeweils Tagungsbände von Symposien und Workshops vor, die jüngst in der von Peter JoR-

1) Aber auch Wien (das Vienna International Centre) war schon zwei Mal Austragungsort von UNGEGNSitzungen, nämlich 2006 und 2011. 


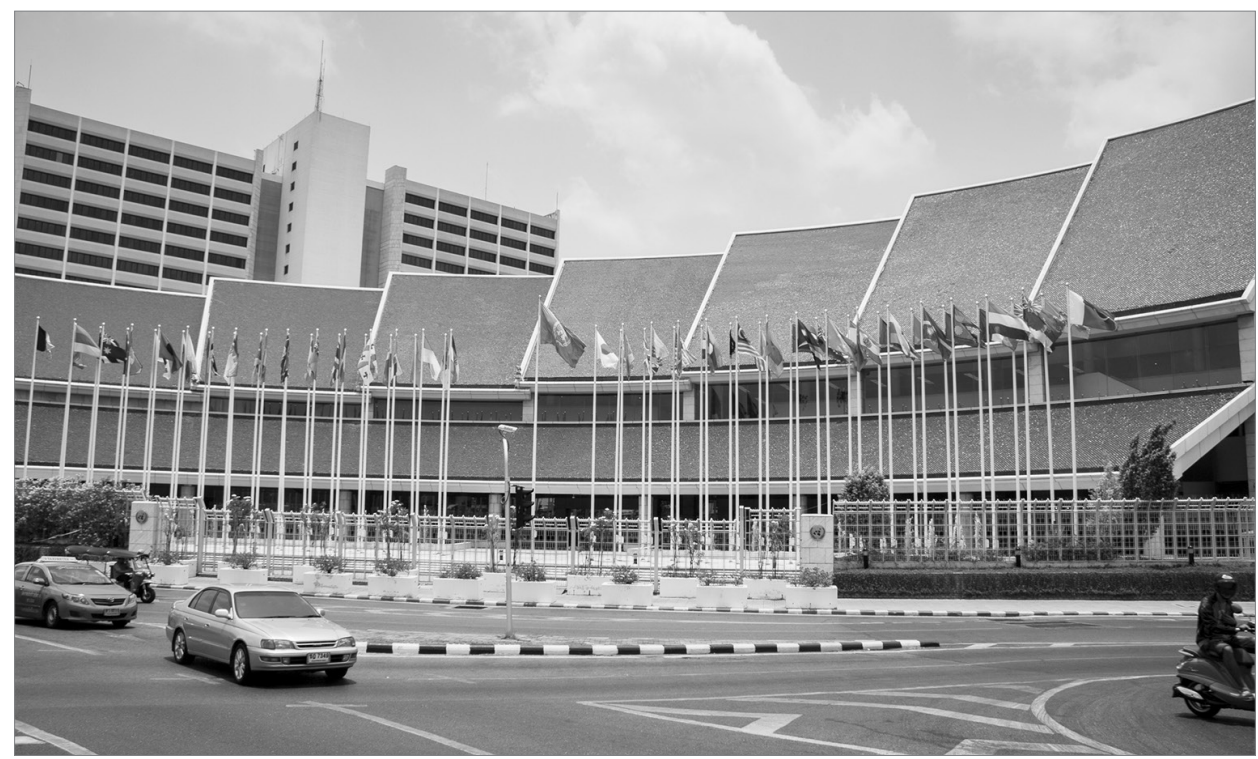

Foto: $\quad$ Maciej ZYCH

Abb. 1: Das Konferenzgebäude der Vereinten Nationen in Bangkok

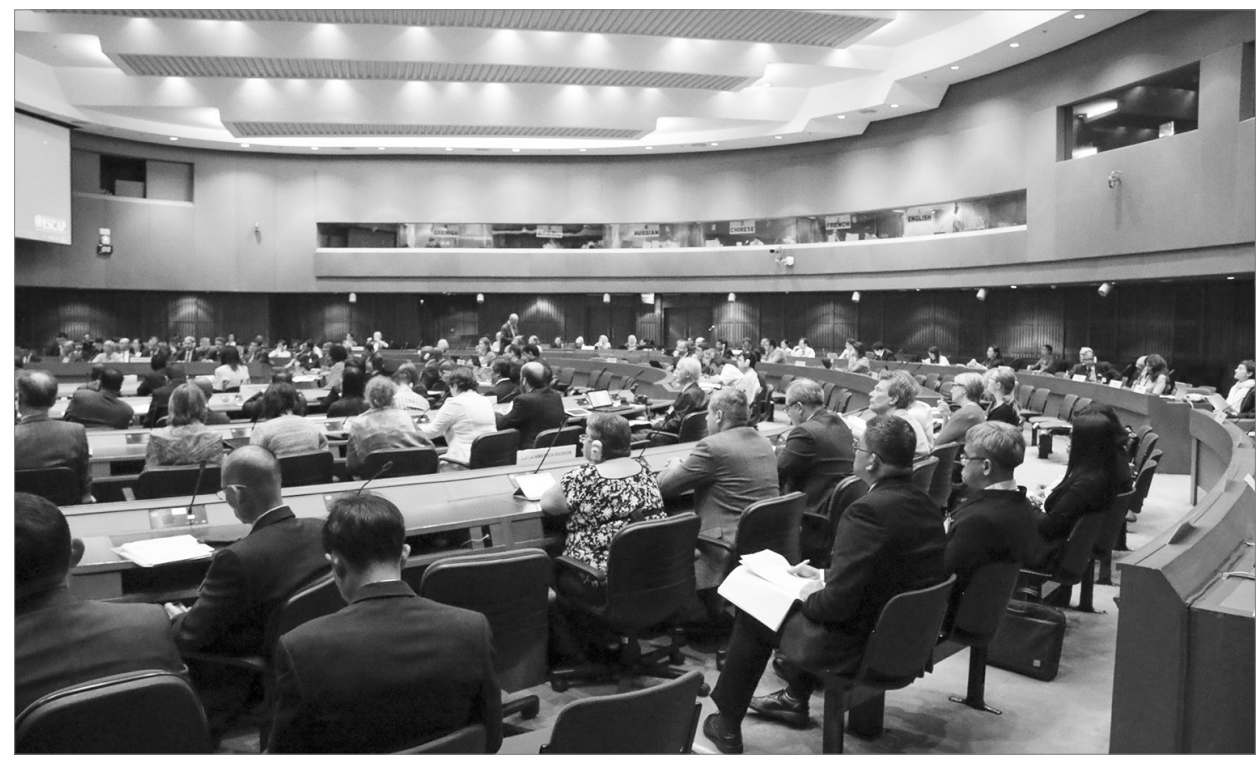

Foto: $\quad$ Maciej ZYCH

Abb. 2: Blick in das Plenum 
DAN und Paul WOODMAN herausgegebenen toponymischen Buchreihe „Name \& Place“ erschienen sind: Band 4 der Reihe mit dem Titel „Confirmation of the Definitions [of the endonym and the exonym]. Proceedings of the $16^{\text {th }}$ UNGEGN Working Group on Exonyms Meeting, Hermagor, 5-7 June 2014“, Band 5 mit dem Titel „Place-Name Changes, Proceedings of the Symposion in Rome, 17-18 November 2014“ und Band 6 mit dem Titel „Criteria for the Use of Exonyms. Proceedings of the $17^{\text {th }}$ UNGEGN Working Group on Exonyms Meeting, Zagreb, 14-16 May 2015“.

Als Convenor der Working Group on Exonyms präsentierte Peter JoRDAN außerdem den Bericht über die Aktivitäten und Ergebnisse dieser Arbeitsgruppe seit der letzten UNGEGN-Sitzung im Jahr 2014. Auch Gerhard RAMPL berichtete als deren internationaler Koordinator über den Stand und die Entwicklung der Toponymic Guidelines, die jeweils für ein Land kurz gefasste Hinweise zur Schreibung geographischer Namen geben sollen.

Mit seinem Vorschlag, den Terminus internationaler Name oder Koinonym für Namen, die von internationalen Behörden und Organen wie der Internationalen Hydrographischen Organisation (IHO) als verbindlich für den internationalen Gebrauch in bestimmten Bereichen (z.B. auf Seekarten) vorgeschrieben werden, zusätzlich zu den Termini Endonym und Exonym einzuführen, löste Peter JoRDAN eine rege Diskussion aus, die in Bangkok aber noch zu keinem endgültigen Ergebnis führte.

Es würde zu weit führen, hier auch noch Arbeitspapiere anderer Länder und Arbeitsgruppen vorzustellen. Erwähnt sei nur die heute zumindest in den größeren Städten Japans, der Republik Korea und Chinas geübte, in der Sitzung vorgestellte und ausführlich diskutierte Praxis, Namen von Verkehrsflächen wie Straßen und Plätzen in zwei Schriften zu bezeichnen: in der jeweils autochthonen ideographischen Schrift und in einer vor allem englisch-phonetischen lateinschriftigen Version, die durchwegs auch die generischen Namenbestandteile (-straße, -platz, etc.) ins Englische übersetzt. Allgemein wurde anerkannt, dass dies ausländischen Touristen die Orientierung wesentlich erleichtert. Es wurden aber auch kritische Fragen gestellt: Was bedeutet das in Bezug auf die ortsbezogene Identität der Bewohner? Kommt man hiermit nicht aus geschäftlichen Gründen Touristen viel weiter entgegen als sprachlichen Minderheiten im eigenen Land, deren Namen zumeist nicht auf Straßentafeln zu finden sind? Trägt es wirklich zur Orientierung bei, wenn solche Namen zwar in Stadtplänen aufscheinen, man Einheimische aber nicht danach fragen kann?

Die Plenarsitzung wurde (fast) vollständig auf YouTube aufgezeichnet und kann von der UNGEGN-Homepage weiterhin abgerufen werden. ${ }^{2)}$

\section{,Side events“6}

UNGEGN-Sitzungen werden aber auch stets für zahlreiche kleinere Treffen von Arbeitsgruppen und Divisions (den nach geographischen oder linguistischen Gesichtspunkten zusammengesetzten Ländergruppen oder Untereinheiten der UNGEGN) ${ }^{3)}$ sowie für Präsentationen und kleine Symposien genützt, die in den Pausen am Rande der Hauptsitzung stattfinden. Der Berichterstatter war an drei solcher Begleitveranstaltungen (side events) aktiv beteiligt: Er leitete das Business Meeting der Working Group on Exonyms (Abb. 3), präsentierte als deren Stellvertretender Vorsitzender die Aktivitäten der Joint IGU/ICA Commission on Toponymy und sprach in einem Symposion zum Thema „Gedenknamen“ (commemorative naming) über die gerade in der AKO in Ausarbeitung befindliche Empfehlung zu Verkehrsflächenbenennungen in Österreich. Es zeigte sich, dass die österreichischen

2) http://unstats.un.org/unsd/geoinfo/UNGEGN/ungegnSession29.html

3) Österreich ist Mitglied der Dutch- and German-speaking Division (DGSD), der auch noch Belgien, Deutschland, die Niederlande, die Schweiz, Südafrika und Suriname angehören. 


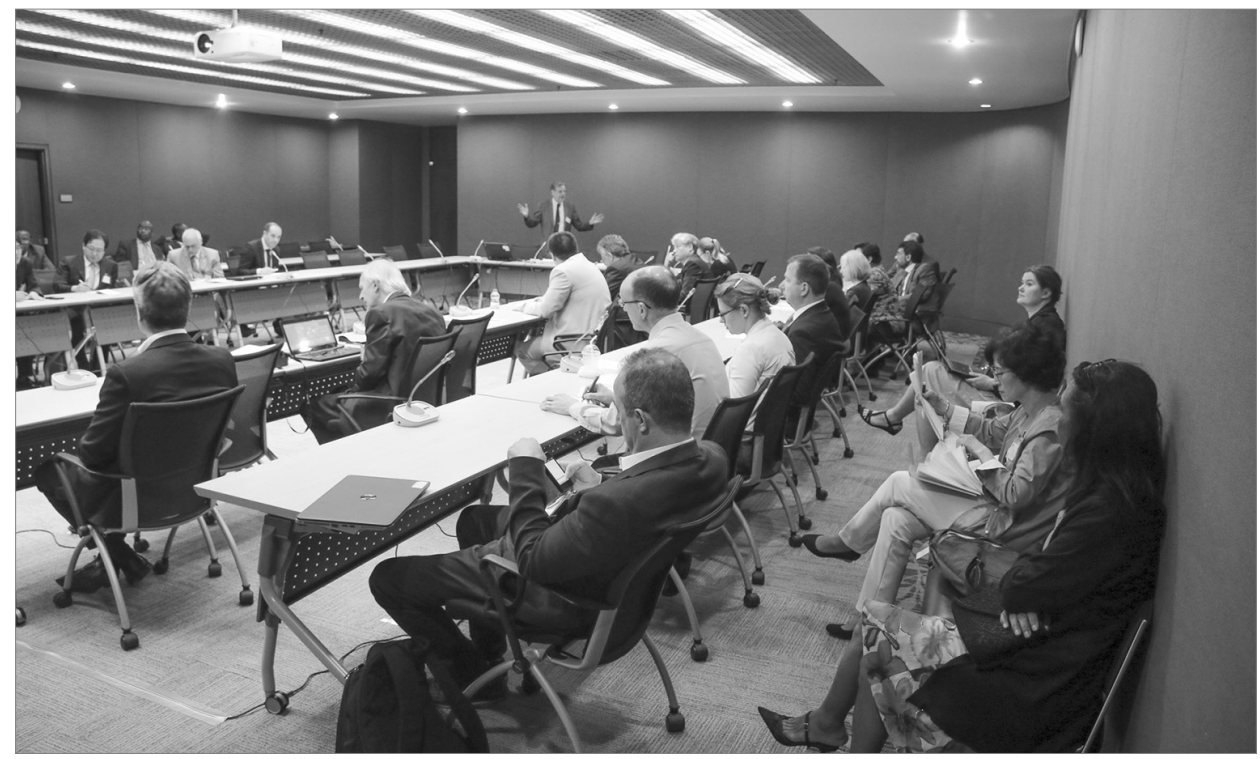

Foto: $\quad$ Maciej ZYCH

Abb. 3: Business meeting der Working Group on Exonyms

Vorschläge in wesentlichen Punkten den schon bestehenden oder geplanten Regelungen in Kanada, Schweden und Finnland, die ebenfalls vorgestellt wurden, entsprechen.

\section{Verhältnis UNGEGN - UN-GGIM}

Ein wichtiger Diskussionspunkt im Plenum, aber auch in zwei eigenen Sitzungen der UNGEGN-Leitung (einschließlich der Convenors) sowie am Rande der Tagung waren das Verhältnis und die möglichen Formen einer Kooperation zwischen UNGEGN und der United Nations Initiative on Global Geospatial Information Management (UN-GGIM). Diese 2009 eingerichtete, ebenfalls dem ECOSOC zugeordnete und aus den Vertretern der staatlichen Karten- und Vermessungsämter (in Österreich des Bundesamtes für Eich- und Vermessungswesen, BEV), also vor allem aus Geodäten und Kartographen bestehende weitere Expertengruppe der Vereinten Nationen beschäftigt sich mit der Gesamtheit der Geoinformationen, damit auch mit geographischen Namen.

Der fachlichen Zusammensetzung dieser Gruppe und ihrer Zielrichtung entsprechend stehen dort allerdings die geodätischen und kartographischen Aspekte von Namen, also deren Orientierungs- und Georeferenzierungsfunktion im Vordergrund. Demgegenüber kann die UNGEGN nicht nur auf eine längere Tradition der Befassung mit geographischen Namen, sondern vor allem auf ihre ganzheitliche Perspektive verweisen, die auch kulturelle und sozialwissenschaftliche Aspekte mit einschließt. Wie schon beschrieben sind es eben diese, die die symbolische Wirkung geographischer Namen im Blickpunkt haben und damit für eine Hauptaufgabe der Vereinten Nationen, die Friedenssicherung, von Bedeutung sind.

Nach anfänglichen Überlegungen, UNGEGN in UN-GGIM einzugliedern, zeichnen sich jetzt Koexistenz und Kooperation der beiden UN-Gremien unter dem gemeinsamen Dach von ECOSOC 
ab. Dazu wird gerade ein „modus cooperandi“ ausgearbeitet, der Möglichkeiten wie regelmäßige Treffen der jeweiligen nationalen Delegierten, regelmäßige Treffen der Leitungen von UNGEGN und UN-GGIM, das Einrichten von Liaisongruppen auf beiden Seiten sowie gemeinsame Gesamtsitzungen umfasst. Er soll von der nächsten Konferenz der Vereinten Nationen zur Standardisierung geographischer Namen, die vom 8. bis 17. August 2017, umrahmt von je einem Tag der 30. UNGEGN-Sitzung, im Hauptquartier der Vereinten Nationen in New York stattfinden wird, beschlossen werden. 